\title{
A Note on Commuting Graphs for Symmetric Groups
}

C. Bates

30 Holtdale Lawn

Holt Park, Leeds LS16 7RP

United Kingdom

chrisjbates@gmail.com

S. Hart

School of Economics, Mathematics \& Statistics

Birkbeck College

Malet Street

London WC1E 7HX

United Kingdom

s.hart@bbk.ac.uk
D. Bundy

Adalbertstrasse 17

24106 Kiel

Germany

davidbundy@yahoo.co.uk

P. Rowley

School of Mathematics

University of Manchester

PO Box 88

Manchester M60 1QD

United Kingdom

peter.j.rowley@manchester.ac.uk

Submitted: May 13, 2008; Accepted: Dec 15, 2008; Published: Jan 7, 2009

Mathematics Subject Classification: 05C25

\begin{abstract}
The commuting graph $\mathcal{C}(G, X)$, where $G$ is a group and $X$ a subset of $G$, has $X$ as its vertex set with two distinct elements of $X$ joined by an edge when they commute in $G$. Here the diameter and disc structure of $\mathcal{C}(G, X)$ is investigated when $G$ is the symmetric group and $X$ a conjugacy class of $G$.
\end{abstract}

\section{Introduction}

The purpose of this note is to record certain properties of commuting graphs $\mathcal{C}(G, X)$ where $G$ is $\operatorname{Sym}(n)$, the symmetric group of degree $n$, and $X$ is a $G$-conjugacy class. In [1] $\mathcal{C}(G, X)$ was investigated when $X$ was a conjugacy class of involutions. There it was shown that $\mathcal{C}(G, X)$ is connected unless $n=2 \ell+1$ or $n=4, \ell=1$ ( $\ell$ being the number of 2-cycles in the involution), and that the diameter of $\mathcal{C}(G, X)$ is at most 3 except for three specifically given graphs (when $n \in\{6,8,10\}$ ). Moreover, if we exclude these three exceptional graphs, an algorithm is given which determines the distance between two vertices using data encoded in $x$-graphs (see Lemma 2.3 and Proposition 3.6 in [1] for more details). For further recent work on commuting graphs we direct the reader to [2], [3] and [4]. 
We recall that $\mathcal{C}(G, X)$ is the graph whose vertex set is $X$ with $x, y \in X(x \neq y)$ joined whenever they commute. Clearly elements of $G$ induce (by conjugation) graph automorphisms of $\mathcal{C}(G, X)$. Since $G$ is transitive on the vertices of $\mathcal{C}(G, X)$, we may without loss choose $a$ to be a fixed element of $X$. Writing $a$ as a product of pairwise disjoint cycles, define $m$ to be the maximum length of a cycle in this decomposition. In view of the work in [1] we shall usually assume that $m$ is greater than two. As compared to [1] (and not surprisingly) we see on perusing Table 1 a great variety of behaviour concerning both connectedness and possible diameters. The information in Table 1 on connectedness and diameters for $n \leq 16$ was obtained with the assistance of the computer algebra package MAGmA [6]. Indeed, the question of when such graphs are connected has been completely answered by Bundy [5].

Before stating our main results we introduce some notation. For $x \in X$ we put $x=\tilde{x} x^{*}$ where $\tilde{x}$ is a cycle of length $m$ and $x^{*}$ is the product of all the remaining disjoint cycles. We assume $G=\operatorname{Sym}(n)(=\operatorname{Sym}(\Omega))$ acts upon the set $\Omega=\{1, \ldots, n\}$. For $g \in G$, $\operatorname{Supp}(g)$ denotes the set of points of $\Omega$ not fixed by $g$ and $\operatorname{Fix}(g)$ the set of points of $\Omega$ fixed by $g$. Set $G^{*}=\operatorname{Sym}(\operatorname{Fix}(\tilde{a}))$. Then $G^{*} \leq G$ and $a^{*} \in G^{*}$. Let $X^{*}$ denote the $G^{*}$-conjugacy class of $a^{*}$. If $q \in \mathbb{Q}$, then $\lceil q\rceil$ means the smallest integer greater than or equal to $q$. We use $d($, $)$ for the standard graph-theoretic distance on $\mathcal{C}(G, X)$ and, for $x \in X$ and $i \in \mathbb{N}$, define the $i^{\text {th }}$ disc of $x$ to be

$$
\Delta_{i}(x)=\{y \in X \mid d(x, y)=i\} .
$$

The diameter of $\mathcal{C}(G, X)$ is denoted by $\operatorname{Diam} \mathcal{C}(G, X)$.

Our first result gives an explicit description of all the discs of $a$ when $a=(12 \cdots m)$ and $n \geq 2 m+1$.

Theorem 1.1. Suppose that $|\operatorname{Fix}(a)|=m+r$ where $m \geq 2$ and $r \geq 1$, and $a=(1 \cdots m)$ $(=\tilde{a})$. For $i$ with $3 \leq i \leq\left\lceil\frac{m-1}{r}\right\rceil+1$, set

$$
\Sigma_{i}(a)=\left\{x \in X\left|\frac{(i-3) r}{2}+1 \leq\right| \operatorname{Supp}(x) \cap \operatorname{Supp}(a) \mid \leq \frac{(i-1) r}{2}\right\}
$$

when $i$ is odd, and

$$
\Sigma_{i}(a)=\left\{x \in X\left|m-\frac{i r}{2} \leq\right| \operatorname{Supp}(x) \cap \operatorname{Supp}(a) \mid \leq m-\frac{(i-2) r}{2}-1\right\}
$$

when $i$ is even. Then

(i) $\Delta_{1}(a)=\{x \in X \mid \operatorname{Supp}(x) \cap \operatorname{Supp}(a)=\emptyset\} \cup(\langle a\rangle \cap X) \backslash\{a\}$;

(ii) $\Delta_{2}(a)=\{x \in X|m-r \leq| \operatorname{Supp}(x) \cap \operatorname{Supp}(a) \mid\} \backslash\left(\Delta_{1}(a) \cup\{a\}\right)$;

(iii) for $i$ with $3 \leq i \leq\left\lceil\frac{m-1}{r}\right\rceil, \Delta_{i}(a)=\Sigma_{i}(a)$; and

(iv) for $i=\left\lceil\frac{m-1}{r}\right\rceil+1, \Delta_{i}(a) \subseteq \Sigma_{i}(a) \subseteq \Delta_{i}(a) \cup \Delta_{i-1}(a)$. 
We next investigate the diameter of $\mathcal{C}(G, X)$ when $a$ has at least $m$ fixed points on $\Omega$.

Theorem 1.2. Let $|\operatorname{Fix}(a)|=m+r$, with $m \geq 3$ and $r \geq 0$.

(i) If $a=(1 \cdots m)(=\tilde{a})$ and $r \geq 1$, then $\operatorname{Diam} \mathcal{C}(G, X)=\left\lceil\frac{m-1}{r}\right\rceil+1$.

(ii) If $r \geq 1$, then $\operatorname{Diam} \mathcal{C}(G, X) \leq m+2 \operatorname{Diam} \mathcal{C}\left(G^{*}, X^{*}\right)$.

(iii) If $r \geq 1$ and $a=(12 \cdots m)(m+1 m+2)$, then $\operatorname{Diam} \mathcal{C}(G, X) \leq\left\lceil\frac{m-1}{r}\right\rceil+3$.

(iv) Suppose that $a=(12 \cdots m)(m+1 \cdots 2 m-1)$. Then $\operatorname{Diam} \mathcal{C}(G, X) \leq 3\left\lceil\frac{m-2}{r+1}\right\rceil+4$.

Our final theorem gives bounds for diameters in the case when $a$ has no fixed points. In order to state this result we define the following function. For $k$ an integer with $k \geq 4$ let

$$
f(k)=\left\{\begin{array}{lll}
2\left(\left(\begin{array}{c}
k-1 \\
2
\end{array}\right)+k-5\right) & \text { if } k \equiv 0 & \bmod 4 \\
2\left(\begin{array}{c}
k-1 \\
2
\end{array}\right)+10 & \text { if } k \equiv 1 & \bmod 4 \\
2\left(\left(\begin{array}{c}
k-1 \\
2
\end{array}\right)+k-4\right) & \text { if } k \equiv 2 & \bmod 4 \\
2\left(\begin{array}{c}
k-1 \\
2
\end{array}\right)+1 & \text { if } k \equiv 3 & \bmod 4
\end{array}\right.
$$

Theorem 1.3. Suppose that $|\operatorname{Fix}(a)|=0, m \geq 4$ and $a$ is the product of $m$ pairwise disjoint cycles each of length $m$. Then Diam $\mathcal{C}(G, X) \leq 3 f(m)+2$.

Note that the case $m=3$ in Theorem 1.3 we have Diam $\mathcal{C}(G, X)=4$ (see Table 1 ). We remark that, as a consequence of Theorems 1 and 2 of [5], all the commuting graphs in Theorems 1.1, 1.2 and 1.3 are connected.

Section 2 is devoted to the proof of Theorem 1.1 while Theorem 1.2 follows from Corollary 2.1, and Propositions 3.3, 3.1 and 3.4; further results which bound $\operatorname{Diam} \mathcal{C}(G, X)$ are given in Propositions 3.2 and 3.5. From Table 1 when $a=(123)(45)$ and $n=9$, $\operatorname{Diam} \mathcal{C}(G, X)=5$ and when $a=(123)(45)$ and $n=8$, Diam $\mathcal{C}(G, X)=7$, so the bounds in Theorem 1.2 ( $i i i)$ and ( $i v)$ are sharp. The bound in part ( $i i)$ of Theorem 1.2 we believe is not best possible. At the end of Section 3 we give the proof of Theorem 1.3.

In Table $1, D$ means that $\mathcal{C}(G, X)$ is disconnected. The entry $d$ (respectively $(d)$ ) means that $\mathcal{C}(G, X)$ is connected and has diameter $d$ (respectively diameter at most $d$ ). 


\begin{tabular}{|c|c|c|c|c|c|c|c|c|c|c|c|c|c|c|}
\hline$a$ & $n=3$ & 4 & 5 & 6 & 7 & 8 & 9 & 10 & 11 & 12 & 13 & 14 & 15 & 16 \\
\hline$\left(\begin{array}{lll}1 & 2 & 3\end{array}\right)$ & 1 & $\mathrm{D}$ & $\mathrm{D}$ & $\mathrm{D}$ & 3 & 2 & 2 & 2 & 2 & 2 & 2 & 2 & 2 & 2 \\
\hline$(1234)$ & - & $\mathrm{D}$ & $\mathrm{D}$ & $\mathrm{D}$ & $\mathrm{D}$ & $\mathrm{D}$ & 4 & 3 & 2 & 2 & 2 & 2 & 2 & 2 \\
\hline$(123)(45)$ & - & - & $\mathrm{D}$ & $\mathrm{D}$ & $\mathrm{D}$ & 7 & 5 & 3 & 3 & 3 & 2 & 2 & 2 & 2 \\
\hline$(12345)$ & - & - & $\mathrm{D}$ & $\mathrm{D}$ & $\mathrm{D}$ & $\mathrm{D}$ & $\mathrm{D}$ & $\mathrm{D}$ & 5 & 3 & 3 & 2 & 2 & 2 \\
\hline$(123)(456)$ & - & - & - & $\mathrm{D}$ & $\mathrm{D}$ & $\mathrm{D}$ & $\mathrm{D}$ & 6 & 4 & 4 & 3 & 3 & 3 & 2 \\
\hline$(1234)(56)$ & - & - & - & $\mathrm{D}$ & $\mathrm{D}$ & $\mathrm{D}$ & $\mathrm{D}$ & 8 & 5 & 4 & 3 & 3 & 3 & 2 \\
\hline$(123456)$ & - & - & - & $\mathrm{D}$ & $\mathrm{D}$ & $\mathrm{D}$ & $\mathrm{D}$ & $\mathrm{D}$ & $\mathrm{D}$ & $\mathrm{D}$ & 6 & 4 & 3 & 3 \\
\hline$(123)(45)(67)$ & - & - & - & - & $\mathrm{D}$ & $\mathrm{D}$ & $\mathrm{D}$ & 6 & 5 & 4 & 3 & 3 & 3 & 3 \\
\hline$(1234)(567)$ & - & - & - & - & $\mathrm{D}$ & $\mathrm{D}$ & $\mathrm{D}$ & $\mathrm{D}$ & 9 & 6 & 5 & 4 & 3 & 3 \\
\hline$(12345)(67)$ & - & - & - & - & $\mathrm{D}$ & $\mathrm{D}$ & $\mathrm{D}$ & $\mathrm{D}$ & $\mathrm{D}$ & 8 & 5 & 4 & 3 & 3 \\
\hline$(1234567)$ & - & - & - & - & $\mathrm{D}$ & $\mathrm{D}$ & $\mathrm{D}$ & $\mathrm{D}$ & $\mathrm{D}$ & $\mathrm{D}$ & $\mathrm{D}$ & $\mathrm{D}$ & 7 & 4 \\
\hline$(123)(456)(78)$ & - & - & - & - & - & $\mathrm{D}$ & $\mathrm{D}$ & $\mathrm{D}$ & 12 & 8 & 5 & 4 & (5) & $(6)$ \\
\hline$(1234)(56)(78)$ & - & - & - & - & - & $\mathrm{D}$ & $\mathrm{D}$ & 12 & 9 & 6 & 5 & $(6)$ & (6) & (6) \\
\hline$(1234)(5678)$ & - & - & - & - & - & $\mathrm{D}$ & $\mathrm{D}$ & $\mathrm{D}$ & $\mathrm{D}$ & 10 & 6 & 5 & 5 & 4 \\
\hline$(12345)(678)$ & - & - & - & - & - & $\mathrm{D}$ & $\mathrm{D}$ & $\mathrm{D}$ & $\mathrm{D}$ & $\mathrm{D}$ & 9 & 6 & 5 & 4 \\
\hline$(12345678)$ & - & - & - & - & - & $\mathrm{D}$ & $\mathrm{D}$ & $\mathrm{D}$ & $\mathrm{D}$ & $\mathrm{D}$ & $\mathrm{D}$ & $\mathrm{D}$ & $\mathrm{D}$ & $\mathrm{D}$ \\
\hline$(123)(456)(789)$ & - & - & - & - & - & - & 4 & $\mathrm{D}$ & $\mathrm{D}$ & 6 & 6 & 5 & $(6)$ & $(7)$ \\
\hline$(1234)(56)(78)(910)$ & - & - & - & - & - & - & - & 7 & $\mathrm{D}$ & 8 & $(9)$ & $(10)$ & $(10)$ & $(9)$ \\
\hline$(1234)(5678)(910)$ & - & - & - & - & - & - & - & $\mathrm{D}$ & $\mathrm{D}$ & $\mathrm{D}$ & $\mathrm{D}$ & $(18)$ & $(14)$ & (11) \\
\hline$(12345)(678910)$ & - & - & - & - & - & - & - & $\mathrm{D}$ & $\mathrm{D}$ & $\mathrm{D}$ & $\mathrm{D}$ & $\mathrm{D}$ & $\mathrm{D}$ & 10 \\
\hline$(123)(456)(789)(101112)$ & - & - & - & - & - & - & - & - & - & 4 & $\mathrm{D}$ & $\mathrm{D}$ & $(10)$ & $(6)$ \\
\hline
\end{tabular}

Table 1: Connectedness and Diameters of $\mathcal{C}(G, X)$ 


\section{Disc Structure for $a=(1 \cdots m)$}

In this section we prove Theorem 1.1. Recall that $a=(1 \cdots m), n=2 m+r$ with $m>2$ and $r \geq 1$. Also we recall the following two sets of vertices which appear in this theorem.

$$
\Sigma_{i}(a)=\left\{x \in X\left|\frac{(i-3) r}{2}+1 \leq\right| \operatorname{Supp}(x) \cap \operatorname{Supp}(a) \mid \leq \frac{(i-1) r}{2}\right\}
$$

when $i$ is odd, and

$$
\Sigma_{i}(a)=\left\{x \in X\left|m-\frac{i r}{2} \leq\right| \operatorname{Supp}(x) \cap \operatorname{Supp}(a) \mid \leq m-\frac{(i-2) r}{2}-1\right\}
$$

when $i$ is even, where $3 \leq i \leq\left\lceil\frac{m-1}{r}\right\rceil+1$.

Proof of Theorem 1.1. It is easy to see that

$$
\Delta_{1}(a)=\{x \in X \mid \operatorname{Supp}(x) \cap \operatorname{Supp}(a)=\emptyset\} \cup(\langle a\rangle \cap X) \backslash\{a\} .
$$

Now consider $x \in \Delta_{2}(a)$. Then $x \in \Delta_{1}(y)$ for some $y \in \Delta_{1}(a)$. Now $x \notin\langle y\rangle$ and $y \notin\langle a\rangle$, for otherwise $x \in \Delta_{1}(a)$. Therefore $\operatorname{Supp}(x) \cap \operatorname{Supp}(y)=\emptyset$ and $\operatorname{Supp}(y) \cap \operatorname{Supp}(a)=\emptyset$. Hence $|\operatorname{Supp}(x) \cap \operatorname{Supp}(a)| \geq m-r$. Conversely, if $|\operatorname{Supp}(x) \cap \operatorname{Supp}(a)| \geq m-r$, then $|\operatorname{Supp}(x) \cup \operatorname{Supp}(a)| \leq m+r$ and there are at least $m$ points which are fixed by $x$ and $a$. Therefore there exists $y$ which commutes with $x$ and $a$. Hence

$$
\Delta_{2}(a)=\{x \in X|m-r \leq| \operatorname{Supp}(x) \cap \operatorname{Supp}(a) \mid\} \backslash\left(\Delta_{1}(a) \cup\{a\}\right) .
$$

If $r \geq m-1$, then we are done. Otherwise, we will prove by induction that for $3 \leq i \leq\left\lceil\frac{m-1}{r}\right\rceil$

$$
\Delta_{i}(a)=\left\{x \in X\left|\frac{(i-3) r}{2}+1 \leq\right| \operatorname{Supp}(x) \cap \operatorname{Supp}(a) \mid \leq \frac{(i-1) r}{2}\right\}
$$

when $i$ is odd, and

$$
\Delta_{i}(a)=\left\{x \in X\left|m-\frac{i r}{2} \leq\right| \operatorname{Supp}(x) \cap \operatorname{Supp}(a) \mid \leq m-\frac{(i-2) r}{2}-1\right\}
$$

when $i$ is even.

We first deal with the case when $i=3$ which will be the basis for the induction. Suppose $x \in \Delta_{3}(a)$ and $y \in \Delta_{1}(x) \cap \Delta_{2}(a)$. If $x \in\langle y\rangle$, then $|\operatorname{Supp}(x) \cap \operatorname{Supp}(a)|=$ $|\operatorname{Supp}(y) \cap \operatorname{Supp}(a)| \geq m-r$, which is a contradiction to $x \in \Delta_{3}(a)$. Hence $\operatorname{Supp}(x) \cap$ $\operatorname{Supp}(y)=\emptyset$ and so $|\operatorname{Supp}(x) \cap \operatorname{Supp}(a)| \leq r$. Also $|\operatorname{Supp}(x) \cap \operatorname{Supp}(a)| \geq 1$, for otherwise $d(x, a) \leq 1$. Conversely, if $1 \leq|\operatorname{Supp}(x) \cap \operatorname{Supp}(a)| \leq r$, then there exists $y \in X$ with $\operatorname{Supp}(x) \cap \operatorname{Supp}(y)=\emptyset$ and $|\operatorname{Supp}(y) \cap \operatorname{Supp}(a)| \geq m-r$. So $d(y, a) \leq 2$ and $y$ commutes with $x$. Hence $x \in \Delta_{3}(a)$ which verifies our assertion in the case when $i=3$. 
Now suppose the induction hypothesis holds for all $3 \leq i \leq k$ and that $x \in \Delta_{k+1}(a)$. Let $y \in \Delta_{1}(x) \cap \Delta_{k}(a)$. If $y \in\langle x\rangle$, then $|\operatorname{Supp}(x) \cap \operatorname{Supp}(a)|=|\operatorname{Supp}(y) \cap \operatorname{Supp}(a)|$ and by the induction hypothesis $x \in \Delta_{k}(a)$, a contradiction. Hence $\operatorname{Supp}(x) \cap \operatorname{Supp}(y)=\emptyset$.

If $k$ is odd, then by the induction hypothesis

$$
\frac{(k-3) r}{2}+1 \leq|\operatorname{Supp}(y) \cap \operatorname{Supp}(a)| \leq \frac{(k-1) r}{2} .
$$

Therefore

$$
|\operatorname{Supp}(y) \cup \operatorname{Supp}(a)| \geq 2 m-\frac{(k-1) r}{2}
$$

and so there are at most $r+\frac{(k-1) r}{2}$ points which are fixed by $y$ and $a$. Hence

$$
m-r-\frac{(k-1) r}{2} \leq|\operatorname{Supp}(x) \cap \operatorname{Supp}(a)| .
$$

But when $|\operatorname{Supp}(x) \cap \operatorname{Supp}(a)| \geq m-\frac{(k-1) r}{2}$, by the induction hypothesis, or when $k=3$, using the result for $\Delta_{2}(a)$, we have that $d(x, a)=k-1$, which is a contradiction. Hence

$$
m-\frac{(k+1) r}{2}=m-r-\frac{(k-1) r}{2} \leq|\operatorname{Supp}(x) \cap \operatorname{Supp}(a)| \leq m-\frac{(k-1) r}{2}-1,
$$

and so $x$ has the required form. Conversely, given $x$ such that $(*)$ holds, by the induction hypothesis it cannot be in any $\Delta_{i}(a)$ for $i$ even and less than $k$. Furthermore, since $k \leq(m-1) / r$,

$$
|\operatorname{Supp}(x) \cap \operatorname{Supp}(a)| \geq m-\frac{(k+1) r}{2} \geq \frac{m+1-r}{2}>\frac{m-1-r}{2} \geq \frac{(k-1) r}{2} .
$$

So again by the induction hypothesis, $x$ cannot be in any $\Delta_{i}(a)$ for $i$ odd and at most $k$. Therefore the distance of $x$ from $a$ is at least $k+1$. It is now easy to see that we can reverse the argument above to show that there exists $y \in \Delta_{k}(a)$ which commutes with $x$. Hence $x \in \Delta_{k+1}(a)$ and this completes the induction step when $k$ is odd.

Now suppose $k$ is even with $x \in \Delta_{k+1}(a)$ and $y \in \Delta_{1}(x) \cap \Delta_{k}(a)$. As before $\operatorname{Supp}(x) \cap$ $\operatorname{Supp}(y)=\emptyset$. Also, by the induction hypothesis

$$
m-\frac{k r}{2} \leq|\operatorname{Supp}(y) \cap \operatorname{Supp}(a)|,
$$

and so $|\operatorname{Supp}(x) \cap \operatorname{Supp}(a)| \leq k r / 2$. If $|\operatorname{Supp}(x) \cap \operatorname{Supp}(a)| \leq \frac{(k-2) r}{2}$, then by the induction hypothesis $d(a, x) \leq k-1$, which is a contradiction. Therefore

$$
\frac{(k-2) r}{2}+1 \leq|\operatorname{Supp}(x) \cap \operatorname{Supp}(a)| \leq \frac{k r}{2},
$$

which is the form required for $x$. Conversely, given $x$ which satisfies these inequalities then by the same argument as in the odd case we can use the criterion $k \leq(m-1) / r$ to 
show that $x$ must be at least distance $k+1$ from $a$ and again it is easy to see that there exists $y \in \Delta_{1}(x) \cap \Delta_{k}(a)$, which completes the induction.

In the case when $i=\left\lceil\frac{m-1}{r}\right\rceil+1$, then the same argument shows that $\Delta_{i}(a) \subseteq \Sigma_{i}(a) \subseteq$ $\Delta_{i}(a) \cup \Delta_{i-1}(a)$, so completing the proof of Theorem 1.1.

We remark that it is possible in Theorem 1.1 to have $\Sigma_{i}(a) \cap \Delta_{i-1}(a) \neq \emptyset$ when $i=\left\lceil\frac{m-1}{r}\right\rceil+1$. For example when $m=4$ and $r=2\left(\right.$ so $\left.\left\lceil\frac{m-1}{r}\right\rceil=2\right)$,

$$
\begin{aligned}
& \Sigma_{3}(a)=\{x \in X|1 \leq| \operatorname{Supp}(x) \cap \operatorname{Supp}(a) \mid \leq 2\} ; \text { and } \\
& \Delta_{2}(a)=\{x \in X|2 \leq| \operatorname{Supp}(x) \cap \operatorname{Supp}(a) \mid\} \backslash\left(\Delta_{1}(a) \cup\{a\}\right) .
\end{aligned}
$$

However, we note that in the case $r=1$ we have $\Sigma_{m}(a)=\Delta_{m}(a)$. Since $X$ is the union of $\{a\}, \Delta_{1}(a), \Delta_{2}(a), \Delta_{3}(a) \ldots, \Delta_{\left\lceil\frac{m-1}{r}\right\rceil+1}(a)$ and $\Delta_{\left\lceil\frac{m-1}{r}\right\rceil+1}(a) \neq \emptyset$, we observe the following corollary of Theorem 1.1.

Corollary 2.1. If $|\operatorname{Fix}(a)|=m+r$ where $m \geq 2, r \geq 1$ and $a=(1 \cdots m)(=\tilde{a})$, then $\operatorname{Diam} \mathcal{C}(G, X)=\left\lceil\frac{m-1}{r}\right\rceil+1$.

\section{Diameter Bounds}

Proposition 3.1. Suppose that $|\operatorname{Fix}(a)| \geq m+1$. Then

$$
\operatorname{Diam} \mathcal{C}(G, X) \leq m+2 \operatorname{Diam} \mathcal{C}\left(G^{*}, X^{*}\right) .
$$

Proof. Let $x\left(=\tilde{x} x^{*}\right) \in X$. Set $\left\{j_{1}, \ldots, j_{k}\right\}=\operatorname{Supp}(\tilde{a}) \backslash \operatorname{Supp}(\tilde{x})$ and let $y \in X$ equal $\tilde{x} y^{*}$ be such that $\left\{j_{1}, \ldots, j_{k}\right\} \subseteq \operatorname{Fix}\left(y^{*}\right)$. Clearly $d(x, y) \leq \operatorname{Diam} \mathcal{C}\left(\operatorname{Sym}\left(\Omega_{1}\right),\left(x^{*}\right)^{\operatorname{Sym}\left(\Omega_{1}\right)}\right)$ where $\Omega_{1}:=\operatorname{Fix}(\tilde{x})$. Let $\Omega_{2}=\operatorname{Fix}\left(y^{*}\right)$. Then $\operatorname{Supp}(\tilde{a}) \subseteq \Omega_{2}$. Note that $\left|\Omega_{2}\right| \geq 2 m+1$. So $d(\tilde{a}, \tilde{x})\left(\operatorname{in} \mathcal{C}\left(\operatorname{Sym}\left(\Omega_{2}\right),(\tilde{a})^{\operatorname{Sym}\left(\Omega_{2}\right)}\right)\right)$ is at most $m$, by Corollary 2.1. Thus $d\left(\tilde{a} y^{*}, \tilde{x} y^{*}\right) \leq m$ and, as before,

$$
d\left(\tilde{a} y^{*}, \tilde{a} a^{*}\right) \leq \operatorname{Diam} \mathcal{C}\left(\operatorname{Sym}(\operatorname{Fix}(\tilde{a})),\left(a^{*}\right)^{\operatorname{Sym}(\operatorname{Fix}(\tilde{a}))}\right)=\operatorname{Diam} \mathcal{C}\left(G^{*}, X^{*}\right) .
$$

Hence $\operatorname{Diam} \mathcal{C}(G, X) \leq m+2 \operatorname{Diam} \mathcal{C}\left(G^{*}, X^{*}\right)$.

The proof of the next result is almost identical to that of Proposition 3.1 and so is omitted.

Proposition 3.2. Suppose $a=(1 \cdots m)(m+1 \cdots m+k) a^{* *}$ where $\{1, \ldots, m+k\} \subseteq$ $\operatorname{Fix}\left(a^{* *}\right)$. Let $\tilde{G}=\operatorname{Sym}\left(\operatorname{Fix}\left(a^{* *}\right)\right)$ and $\tilde{X}$ be the $\tilde{G}$-conjugacy class of $(1 \cdots m)(m+1 \cdots m+$ $k$ ). If $k<m$ and $|\operatorname{Fix}(a)|=m$, then

$$
\operatorname{Diam} \mathcal{C}(G, X) \leq \operatorname{Diam} \mathcal{C}(\tilde{G}, \tilde{X})+2 \operatorname{Diam} \mathcal{C}\left(G^{*}, X^{*}\right) .
$$

Proposition 3.3. Let $a=(12 \cdots m)(m+1 m+2)$ have $m+r$ fixed points, for $m \geq 3$ and $r \geq 1$. Then $\operatorname{Diam} \mathcal{C}(G, X) \leq\left\lceil\frac{m-1}{r}\right\rceil+3$. 
Proof. Let $x \in X$ have $m$-cycle $\tilde{x}$, and define $s=|\operatorname{Supp}(\tilde{a}) \cap \operatorname{Supp}(\tilde{x})|$. Then

$$
|\Omega \backslash(\operatorname{Supp}(\tilde{a}) \cup \operatorname{Supp}(\tilde{x}))| \geq s+3 .
$$

We consider separately the cases $s=0, s=1$ and $s \geq 2$.

Assume that $s=0$. Then let $y=(12 \cdots m)(\alpha \beta)$ where $\alpha, \beta \notin \operatorname{Supp}(\tilde{a}) \cup \operatorname{Supp}(\tilde{x})$ and either $(\alpha \beta)=(m+1 \quad m+2)$ or $\{\alpha, \beta\} \cap\{m+1, m+2\}=\emptyset$. Since $\mid \Omega \backslash(\operatorname{Supp}(\tilde{a}) \cup$ $\operatorname{Supp}(\tilde{x})) \mid \geq 3$ at least one of these is possible. Now let $z=\tilde{x}(\alpha \beta)$. Then $[a, y]=[y, z]=1$ and $d(z, x) \leq 2$. Hence $d(a, x) \leq 4 \leq\left\lceil\frac{m-1}{r}\right\rceil+3$.

Now assume that $s=1$. Suppose first that $\{m+1, m+2\} \cap \operatorname{Supp}(\tilde{x}) \neq \emptyset$. Let $(\gamma \delta)$ be the 2-cycle in $x$. If $\{m+1, m+2\} \subseteq \operatorname{Supp}(\tilde{x})$, then let $\alpha, \beta$ be such that $\{\alpha, \beta\} \cap$ $(\operatorname{Supp}(\tilde{a}) \cup \operatorname{Supp}(\tilde{x}) \cup\{\gamma, \delta\})=\emptyset$. If $|\{m+1, m+2\} \cap \operatorname{Supp}(\tilde{x})|=1$, then let $\alpha$, $\beta$ be such that either $\{\alpha, \beta\}=\{\gamma, \delta\}$ (if $\{\gamma, \delta\} \cap(\operatorname{Supp}(\tilde{a}) \cup\{m+1, m+2\})=\emptyset$ ) or $\{\alpha, \beta\} \cap[(\{\gamma, \delta, m+1, m+2\}) \cup \operatorname{Supp}(\tilde{a}) \cup \operatorname{Supp}(\tilde{x})]=\emptyset$. This is possible because $|\Omega \backslash(\operatorname{Supp}(\tilde{a}) \cup \operatorname{Supp}(\tilde{x}) \cup\{m+1, m+2\})| \geq 3$. In either case let $y=\tilde{a}(\alpha \beta)$. Then $[a, y]=1$. Let $z=\tilde{x}(\alpha \beta)$. Then $d(y, z) \leq\left\lceil\frac{m-1}{r}\right\rceil+1$ by Corollary 2.1 and $[z, x]=1$. Hence $d(a, x) \leq\left\lceil\frac{m-1}{r}\right\rceil+3$. Now we are left with the case $\{m+1, m+2\} \cap \operatorname{Supp}(\tilde{x})=\emptyset$. Set $y=\tilde{x}(m+1 m+2)$. Then, again by Corollary $2.1, d(a, y) \leq\left\lceil\frac{m-1}{r}\right\rceil+1$, and $d(y, x) \leq 2$. So $d(a, x) \leq\left\lceil\frac{m-1}{r}\right\rceil+3$.

Finally assume that $s \geq 2$. Suppose $\{m+1, m+2\} \cap \operatorname{Supp}(\tilde{x}) \neq \emptyset$. Then $\mid \Omega \backslash(\operatorname{Supp}(\tilde{a}) \cup$ $\operatorname{Supp}(\tilde{x}) \cup\{m+1, m+2\}) \mid \geq s+2 \geq 4$. So let $\{\alpha, \beta\} \subseteq \Omega \backslash(\operatorname{Supp}(\tilde{a}) \cup \operatorname{Supp}(\tilde{x}) \cup\{m+$ $1, m+2, \gamma, \delta\})$ where $\{\gamma, \delta\}$ is the 2-cycle for $x$. Let $y=\tilde{a}(\alpha \beta)$ and $z=\tilde{x}(\alpha \beta)$. Then $[a, y]=[x, z]=1$ and $d(y, z) \leq\left\lceil\frac{m-1}{r}\right\rceil+1$ by Corollary 2.1. So $d(a, x) \leq\left\lceil\frac{m-1}{r}\right\rceil+3$. If $\{m+1, m+2\} \cap \operatorname{Supp}(\tilde{x})=\emptyset$, then $y=\tilde{x}(m+1 m+2)$. Using Corollary 2.1, $d(a, y) \leq\left\lceil\frac{m-1}{r}\right\rceil+1$. Hence, as $d(y, x) \leq 2, d(a, x) \leq\left\lceil\frac{m-1}{r}\right\rceil+3$ and the result is proven.

Proposition 3.4. Let $a=(12 \cdots m)(m+1 \cdots 2 m-1)$ have $m+r$ fixed points, for $m \geq 3$ and $r \geq 0$. Then $\operatorname{Diam} \mathcal{C}(G, X) \leq 3\left\lceil\frac{m-2}{r+1}\right\rceil+4$.

We remark that this bound is strict - if $a=(123)(45) \in S_{8}$ then $\operatorname{Diam} \mathcal{C}(G, X)=7=$ $3 \times 1+4$.

Proof. Let $\tilde{x}=\left(\alpha_{1} \cdots \alpha_{m}\right)$, and define $k=|\operatorname{Supp}(\tilde{a}) \cap \operatorname{Supp}(\tilde{x})|$. We will consider various possibilities for $k$.

Firstly, suppose $k \leq r$. Let $a_{1}=\tilde{a}_{1}(m+1 \cdots 2 m-1)$ and $a_{2}=\tilde{a}_{2}(m+1 \cdots 2 m-1)$, where the $m$-cycles $\tilde{a}_{1}$ and $\tilde{a}_{2}$ are chosen such that $\operatorname{Supp}(\tilde{x}) \backslash \operatorname{Supp}(a) \subseteq \operatorname{Supp}\left(\tilde{a}_{1}\right) \subseteq \Omega \backslash \operatorname{Supp}(a)$ and $\operatorname{Supp}(\tilde{x}) \cup \operatorname{Supp}\left(a_{1}\right) \subseteq \operatorname{Fix}\left(\tilde{a}_{2}\right)$. In particular, $\left|\operatorname{Supp}\left(\tilde{a}_{2}\right) \cap \operatorname{Supp}(\tilde{x})\right|=0$. Then $\left[a, a_{1}\right]=$ $\left[a_{1}, a_{2}\right]=1$ and hence $d\left(a, a_{2}\right) \leq 2$. Now there exists $x_{1} \in X$ with $d\left(a_{2}, x_{1}\right) \leq\left\lceil\frac{m-2}{r+1}\right\rceil+1$ such that $x_{1}=\tilde{a}_{2}\left(\beta_{1} \cdots \beta_{m-1}\right)$ where $\operatorname{Supp}(\tilde{x}) \subseteq \operatorname{Fix}\left(x_{1}\right)$ by Corollary 2.1. Hence, for $x_{2}=\left(\alpha_{1} \cdots \alpha_{m}\right)\left(\beta_{1} \cdots \beta_{m-1}\right),\left[x_{1}, x_{2}\right]=1$ and again $d\left(x_{2}, x\right) \leq\left\lceil\frac{m-2}{r+1}\right\rceil+1$. Therefore $d(a, x) \leq 2\left(\left\lceil\frac{m-2}{r+1}\right\rceil+1\right)+3<3\left\lceil\frac{m-2}{r+1}\right\rceil+4$.

Now assume that $r<k<m-1$. Then $\alpha_{i_{k+1}}, \ldots, \alpha_{i_{m}}>m$ for some $i_{k+1}, \ldots, i_{m}$. So there exists $x_{1}=(1 \cdots m)\left(\beta_{1} \cdots \beta_{m-1}\right) \in X$ with $\left\{\alpha_{i_{k+1}}, \ldots, \alpha_{i_{m}}\right\}=\left\{\beta_{k}, \ldots, \beta_{m-1}\right\}$. 
Let $l=\left|\left\{\beta_{1} \cdots \beta_{m-1}\right\} \cap\{m+1 \cdots 2 m-1\}\right|$. By varying the size of $\left\{\beta_{1}, \ldots, \beta_{k-1}\right\} \cap$ $\{m+1, \ldots, 2 m-1\}$ we may choose $k$ different values for $l$. But by Theorem 1.1, at most $r$ of these values can occur for elements of $\Sigma_{\left\lceil\frac{m-2}{r+1}\right\rceil+1}(a)$, and so, since $k>r$, we may choose $x_{1}$ so that $d\left(a, x_{1}\right) \leq\left\lceil\frac{m-2}{r+1}\right\rceil$. Now define $x_{2}=\left(\gamma_{1} \cdots \gamma_{m}\right)\left(\beta_{1} \cdots \beta_{m-1}\right)$ for some $\gamma_{1}, \ldots, \gamma_{m}>m$. Clearly $x_{1} x_{2}=x_{2} x_{1}$. Now $d\left(x_{2}, x_{3}\right)=\left\lceil\frac{m-2}{r+1}\right\rceil+1$ where $x_{3}$ has $m$-cycle $\left(\gamma_{1} \cdots \gamma_{m}\right)$ and fixes $\left(\alpha_{1} \cdots \alpha_{m}\right)$. Hence $d\left(x_{3}, x\right) \leq\left(\left\lceil\frac{m-2}{r+1}\right\rceil+1\right)+1$. So, recalling that $d\left(a, x_{1}\right) \leq\left\lceil\frac{m-2}{r+1}\right\rceil, d\left(x_{1}, x_{2}\right)=1$ and $d\left(x_{2}, x_{3}\right) \leq\left\lceil\frac{m-2}{r+1}\right\rceil+1$, we conclude that $d(a, x) \leq 3\left\lceil\frac{m-2}{r+1}\right\rceil+4$.

If $k=m$, then let $x_{1}=(m+1 \cdots 2 m-1)(2 m \cdots 3 m-1)$ and $x_{2}=\left(\alpha_{1} \cdots \alpha_{m}\right)(m+$ $1 \cdots 2 m-1)$, noting that in this case $\left\{\alpha_{1}, \ldots, \alpha_{m}\right\}=\{1, \ldots, m\}$. It is clear that $a x_{1}=x_{1} a$, $x_{1} x_{2}=x_{2} x_{1}$ and $d\left(x_{2}, x\right) \leq\left\lceil\frac{m-2}{r+1}\right\rceil+1$. Thus $d(a, x) \leq\left\lceil\frac{m-2}{r+1}\right\rceil+3$.

Finally we deal with the case $k=m-1$. Using the notation above, $\alpha_{i_{1}} \notin\{1, \ldots, m\}$ but $\alpha_{i_{j}} \in\{1, \ldots, m\}$ for each $j>1$. Set $x_{1}=(1 \cdots m)\left(\alpha_{i_{2}} \cdots \alpha_{i_{m}}\right)$ and, for some $\gamma_{j}>m$, $x_{2}=\left(\gamma_{1} \cdots \gamma_{m}\right)\left(\alpha_{i_{2}} \cdots \alpha_{i_{m}}\right)$. Let $x_{3}$ be such that $x_{3}$ has $m$-cycle $\left(\gamma_{1} \cdots \gamma_{m}\right)$ and fixes $\alpha_{1}, \ldots, \alpha_{m}$. Now let $x_{4}$ have $m$-cycle $\left(\alpha_{1} \cdots \alpha_{m}\right)$ and fix $\gamma_{1}, \ldots, \gamma_{m}$. Then we see that $d\left(a, x_{1}\right) \leq\left\lceil\frac{m-2}{r+1}\right\rceil+1, d\left(x_{1}, x_{2}\right)=1, d\left(x_{2}, x_{3}\right)=1, d\left(x_{3}, x_{4}\right)=1$, and $d\left(x_{4}, x\right) \leq\left\lceil\frac{m-2}{r+1}\right\rceil+1$. Therefore $d(a, x) \leq 2\left\lceil\frac{m-2}{r+1}\right\rceil+5 \leq 3\left\lceil\frac{m-2}{r+1}\right\rceil+4$ (since $m \geq 3$ ). We have shown, for all possible values of $k$, that $d(a, x) \leq 3\left\lceil\frac{m-2}{r+1}\right\rceil+4$ and so the proposition is proved.

Proposition 3.5. Let $a=(1 \ldots m)(m+1 \ldots m+k)=\tilde{a} a^{*}$, where $k<m$, and assume $|\operatorname{Fix}(a)|=r+m \geq m$. Then

$$
\operatorname{Diam} \mathcal{C}(G, X) \leq\left(\left\lceil\frac{k-1}{m+r-k}\right\rceil+2\right)\left(\left\lceil\frac{m-1}{k+r}\right\rceil+2\right)-1 .
$$

Proof. For $x \in X$ let $G_{x}^{*}=\operatorname{Sym}(\operatorname{Fix}(\tilde{x}))$ and let $X_{x}=x^{G_{x}^{*}}$. Consider the graph $\Gamma$ with vertices $\left\{X_{x} \mid x \in X\right\}$ and edges $\left\{X_{x}, X_{y}\right\}$, for $x, y \in X$, whenever there exist $v \in X_{x}$ and $w \in X_{y}$ such that $v w=w v$. Then Diam $\Gamma=\operatorname{Diam} \mathcal{C}\left(G,(\tilde{a})^{G}\right)$. Now let $\pi$ be a path of minimum length in $\mathcal{C}(G, X)$ between two points in $X$. Then we can obtain a path $\pi_{\Gamma}$ in $\Gamma$ by taking $X_{x}$ for each $x \in \pi$ and then contracting an edge whenever neighbours $x$ and $y$ in $\pi$ are such that $X_{x}=X_{y}$. We have that the length of $\pi_{\Gamma}$ is at most Diam $\Gamma$ and at each vertex of $\pi_{\Gamma}$ at most $\operatorname{Diam} \mathcal{C}\left(G^{*}, X^{*}\right)$ edges of $\pi$ were contracted. Hence, using Corollary 2.1,

$$
\begin{aligned}
|\pi| & \leq\left|\pi_{\Gamma}\right|+\left(\operatorname{Diam} \mathcal{C}\left(G^{*}, X^{*}\right)\right)(\operatorname{Diam} \Gamma+1) \\
& =\left(\operatorname{Diam} \mathcal{C}\left(G^{*}, X^{*}\right)+1\right)\left(\operatorname{Diam}\left(G,\left(\tilde{a}^{G}\right)+1\right)\right)-1 \\
& =\left(\left\lceil\frac{k-1}{m+r-k}\right\rceil+2\right)\left(\left\lceil\frac{m-1}{k+r}\right\rceil+2\right)-1 .
\end{aligned}
$$

The final part of this section is devoted to the proof of Theorem 1.3. From now on we shall assume that $n=m^{m}, m \geq 4$ and $a$ is the product of $m$ pairwise disjoint cycles each of length $m$.

Given a cycle $c=\left(\alpha_{1} \ldots \alpha_{m}\right)$, we say that an $m$-cycle $c^{\prime}$ differs from $c$ by a simple transposition if $c^{\prime}$ is $c$ with two adjacent elements interchanged. For example, if $c=(1234)$, then (1342), (1324), (1243) and (1423) all differ from $c$ by a simple transposition. 
Lemma 3.6. Let $x=\prod_{i=1}^{m} x_{i} \in X$ where each $x_{i}$ is an $m$-cycle and the $x_{i}$ are pairwise disjoint. Suppose $y=\prod_{i=1}^{m} y_{i}$ where $y_{i}$ differs from $x_{i}$ by a simple transposition. Then $d(x, y)=2$.

Proof. For each $i$, write $x_{i}=\left(x_{i, 1} x_{i, 2} x_{i, 3} \cdots x_{i, m}\right)$ where $x_{i, 1}$ is chosen so that $y_{i}=\left(x_{i, 2} x_{i, 1} x_{i, 3} \cdots x_{i, m}\right)$. Now define $z \in X$ as follows.

$$
z=\left(x_{1,1} x_{2,1} \cdots x_{m, 1}\right) \cdots\left(x_{1, m} x_{2, m} \cdots x_{m, m}\right) .
$$

Then $d(x, z)=1=d(y, z)$. Therefore $d(x, y)=2$.

Corollary 3.7. Suppose that $x=\prod_{i=1}^{m} x_{i}$ and $y=\prod_{i=1}^{m} y_{i}$ are elements of $X$ and that, for $1 \leq i \leq m, y_{i}$ differs from $x_{i}$ by an even number $n_{i}$ of transpositions. Let $N=\max \left\{n_{i}\right\}$. Then $d(x, y) \leq 2 N$.

Note that the identity permutation is an even permutation, hence any cycle differs from itself by an even number of transpositions. We may therefore permute elements of individual cycles if necessary without affecting other cycles, as long as the permutation in question is even. The next result shows that we can guarantee this if $m$ is even.

In the proofs of Propositions 3.8 and 3.9, the permutations appearing there are acting upon the 'positions'; that is to say (23) applied to an $m$-cycle $c$ in $\operatorname{Sym}\{1,2, \ldots, m\}$ means 'interchange the second and third element of $c$ ' and not 'interchange the numbers 2 and 3'. To illustrate this we give an example of the instance in Proposition 3.8 where $(i i+1)$ is replaced by

$$
(i+1 i+2) \cdots(m-1 m)(m 1)(12) \cdots(i-2 i-1) .
$$

Suppose $m=6,(i i+1)=(45)$ and $c=(123456)$. Applying (56)(61)(12)(23) to (123456) means

$$
(123456) \stackrel{(56)}{\longrightarrow}(123465) \stackrel{(61)}{\longrightarrow}(523461) \stackrel{(12)}{\longrightarrow}(253461) \stackrel{(23)}{\longrightarrow}(235461)(=(123546)=d) .
$$

Proposition 3.8. Suppose $m$ is even. Let $c$ and $d$ be $m$-cycles with $\operatorname{Supp}(c)=\operatorname{Supp}(d)$. Then $c$ and d differ by an even number $N$ of transpositions and

$$
N \leq\left\{\begin{array}{ccc}
\left(\begin{array}{c}
m-1 \\
2
\end{array}\right)+m-5 & \text { if } m \equiv 0 \quad \bmod 4 \\
\left(\begin{array}{c}
m-1 \\
2
\end{array}\right)+m-4 & \text { if } m \equiv 2 \quad \bmod 4
\end{array}\right.
$$

Proof. Clearly $d$ may be obtained from $c$ by a permutation $\sigma$ of $\operatorname{Sym}(2, \ldots, m$ ) (note that for example (23) means 'interchange the second and third elements of $c$ ', rather than 'interchange the numbers 2 and 3'). Now $\operatorname{Sym}(2, \ldots, m)$ is generated by the simple transpositions $(23),(34), \ldots,(m-1 m)$ and the longest such expression is length $\left(\begin{array}{c}m-1 \\ 2\end{array}\right)$; this corresponds to sending $23 \cdots m$ to $m \cdots 32$, in other words $c$ to $c^{-1}$. If the length $L$ of $\sigma$ is even, then $N=L$ and we are done. Otherwise, suppose $(i i+1)$ is the final transposition of $\sigma$. Then replace $(i i+1)$ with $(i+1 i+2) \cdots(m-1 m)(m 1)(12) \cdots(i-2 i-1)$ (which still has the effect of interchanging the $i$ and $(i+1)^{\text {st }}$ elements of the cycle). Then 
$N=L-1+m-2=L+m-3$, which is even. Suppose $\left(\begin{array}{c}m-1 \\ 2\end{array}\right)$ is even (that is, $m \equiv 2$ $\bmod 4)$. Then $N \leq\left(\begin{array}{c}m-1 \\ 2\end{array}\right)-1+m-3=\left(\begin{array}{c}m-1 \\ 2\end{array}\right)+m-4$. Suppose $\left(\begin{array}{c}m-1 \\ 2\end{array}\right)$ is odd. If $d=c^{-1}$, then we may convert $c$ into $d$ by the transposition $(1 \mathrm{~m})$ followed by the permutation of $\operatorname{Sym}(2 \cdots m-1)$ which reverses the order of the elements. This has length $\left(\begin{array}{c}m-2 \\ 2\end{array}\right)$. Hence $N \leq \max \left\{\left(\begin{array}{c}m-2 \\ 2\end{array}\right)+1,\left(\left(\begin{array}{c}m-1 \\ 2\end{array}\right)-2\right)+m-3\right\}$ corresponding to the cases $d=c^{-1}$ and $d \neq c^{-1}$. Therefore $N \leq\left(\begin{array}{c}m-1 \\ 2\end{array}\right)+m-5$ in the case $m \equiv 0 \bmod 4$.

Proposition 3.9. Suppose that $m$ is odd. If $x, y \in X$ have the same orbits, then there exists $x^{\prime} \in X$ with the same orbits as $x$ and $y$ such that

(a) cycles of $x^{\prime}$ and $y$ with the same orbit differ by an even number, not more than $\left(\begin{array}{c}m-1 \\ 2\end{array}\right)$, of simple transpositions;

(b) $d\left(x, x^{\prime}\right) \leq 1$ if $m \equiv 3 \bmod 4$ and $d\left(x, x^{\prime}\right) \leq 10$ if $m \equiv 1 \bmod 4$.

Proof. Suppose $m \equiv 3 \bmod 4$. Given a cycle $x_{i}$ of $x, x_{i}$ differs from $x_{i}^{-1}$ by an odd number of transpositions (and hence an odd number of simple transpositions) of $\operatorname{Sym}(2,3, \ldots, m)$. Therefore choosing $x_{i}^{\prime}$ to be $x_{i}$ or $x_{i}^{-1}$ as appropriate means that the corresponding cycle of $y$ differs from $x_{i}^{\prime}$ by an even number $N$ of simple transpositions of $\operatorname{Sym}(2,3 \ldots, m)$ and hence $N \leq\left(\begin{array}{c}m-1 \\ 2\end{array}\right)$. Since $x_{i}$ commutes with $x_{i}^{\prime}$ for all cycles $x_{i}$ of $x$ we see that $d\left(x, x^{\prime}\right) \leq 1$. Next we consider the case $m \equiv 1 \bmod 4$. Write $x=x_{1} x_{2} \cdots x_{m}$ and $y=y_{1} \cdots y_{m}$, where $\operatorname{Supp}\left(x_{i}\right)=\operatorname{Supp}\left(y_{i}\right)$ for $1 \leq i \leq m$. Suppose that $x_{i}$ and $y_{i}$ differ by an odd number of simple transpositions for $1 \leq i \leq t$ and by an even number of simple transpositions for $i>t$. We assume first that $t=2 k$ is even. We will describe elements $b, c, d, e, x^{\prime}$ of $X$. It is helpful to write each one as an $m \times m$ array, where the rows are the cycles.

$$
\begin{aligned}
\left.x=\left(\begin{array}{llll}
x_{1,1} & x_{1,2} & \cdots & x_{1, m} \\
x_{2,1} & x_{2,2} & \cdots & x_{2, m} \\
\vdots & \vdots & & \vdots \\
x_{m, 1} & x_{m, 2} & \cdots & x_{m, m}
\end{array}\right) ; \quad \begin{array}{llllll}
x_{1,1} & x_{2,1} & \cdots & x_{m, 1} \\
x_{1,2} & x_{2,2} & \cdots & x_{m, 2} \\
\vdots & \vdots & & \vdots \\
x_{1, m} & x_{2, m} & \cdots & x_{m, m}
\end{array}\right) \\
c=\left(\begin{array}{llllll}
x_{1,1} & x_{1,2} & x_{3,3} & x_{3,4} & \cdots & x_{3, m} \\
x_{2,1} & x_{2,2} & x_{4,3} & x_{4,4} & \cdots & x_{4, m} \\
\vdots & \vdots & \vdots & \vdots & & \vdots \\
x_{m, 1} & x_{m, 2} & x_{2,3} & x_{2,4} & \cdots & x_{2, m}
\end{array}\right)
\end{aligned}
$$

Next we permute the first $k$ rows of $c$ using the double transposition (12)(34) to produce $d$.

$$
d=\left(\begin{array}{lllllll}
x_{1,2} & x_{1,1} & x_{3,4} & x_{3,3} & x_{3,5} & \cdots & x_{3, m} \\
\vdots & \vdots & \vdots & \vdots & \vdots & & \vdots \\
x_{k, 2} & x_{k, 1} & x_{k+2,4} & x_{k+2,3} & x_{k+2,5} & \cdots & x_{k+2, m} \\
x_{k+1,1} & x_{k+1,2} & x_{k+3,3} & x_{k+3,4} & x_{k+3,5} & \cdots & x_{k+3, m} \\
\vdots & \vdots & \vdots & \vdots & \vdots & & \vdots \\
x_{m, 1} & x_{m, 2} & x_{2,3} & x_{2,4} & x_{2,5} & \cdots & x_{2, m}
\end{array}\right)
$$




$$
e=d^{T}=\left(\begin{array}{llllllll}
\mathbf{x}_{\mathbf{1 , 2}} & \cdots & x_{k, 2} & x_{k+1,1} & \cdots & x_{m-2,1} & x_{m-1,1} & x_{m, 1} \\
\mathbf{x}_{\mathbf{1 , 1}} & \cdots & x_{k, 1} & x_{k+1,2} & \cdots & x_{m-2,2} & x_{m-1,2} & x_{m, 2} \\
x_{3,4} & \cdots & x_{k+2,4} & x_{k+3,3} & \cdots & x_{m, 3} & \mathbf{x}_{\mathbf{1}, \mathbf{3}} & x_{2,3} \\
x_{3,3} & \cdots & x_{k+2,3} & x_{k+3,4} & \cdots & x_{m, 4} & \mathbf{x}_{\mathbf{1 , 4}} & x_{2,4} \\
x_{3,5} & \cdots & x_{k+2,5} & x_{k+3,5} & \cdots & x_{m, 5} & \mathbf{x}_{\mathbf{1 , 5}} & x_{2,5} \\
\vdots & & \vdots & \vdots & & \vdots & \vdots & \vdots \\
x_{3, m} & \cdots & x_{k+2, m} & x_{k+3, m} & \cdots & x_{m, m} & \mathbf{x}_{\mathbf{1 , m}} & x_{2, m}
\end{array}\right)
$$

The bold entries of $e$ form the first cycle (row) of $x^{\prime}$ below. The second cycle consists of the entries immediately to the right of the bold entries, and so on.

$$
x^{\prime}=\left(\begin{array}{lllllll}
x_{1,2} & x_{1,1} & x_{1,3} & x_{1,4} & x_{1,5} & \cdots & x_{1, m} \\
\vdots & \vdots & \vdots & \vdots & \vdots & & \vdots \\
x_{k, 2} & x_{k, 1} & x_{k, 3} & x_{k, 4} & x_{k, 5} & \cdots & x_{k, m} \\
x_{k+1,1} & x_{k+1,2} & x_{k+1,4} & x_{k+1,3} & x_{k+1,5} & \cdots & x_{k+1, m} \\
\vdots & \vdots & \vdots & \vdots & \vdots & & \vdots \\
x_{2 k, 1} & x_{2 k, 2} & x_{2 k, 4} & x_{2 k, 3} & x_{2 k, 5} & \cdots & x_{2 k, m} \\
x_{2 k+1,1} & x_{2 k+1,2} & x_{2 k+1,3} & x_{2 k+1,4} & x_{2 k+1,5} & \cdots & x_{2 k+1, m} \\
\vdots & \vdots & \vdots & \vdots & \vdots & & \vdots \\
x_{m, 1} & x_{m, 2} & x_{m, 3} & x_{m, 4} & x_{m, 5} & \cdots & x_{m, m}
\end{array}\right)
$$

Now $d(x, b)=1=d(b, c)$. By Corollary $3.7 d(c, d) \leq 4$. Moreover $d(d, e)=1=d\left(e, x^{\prime}\right)$. Hence $d\left(x, x^{\prime}\right) \leq 8$. Note that each of the first $t$ cycles of $x^{\prime}$ differs from the corresponding cycle of $x$ by one simple transposition (either (12) or (34)). Hence for $1 \leq i \leq m, x_{i}^{\prime}$ differs from $y_{i}$ by an even number of simple transpositions, so $x^{\prime}$ is as required.

Suppose $t$ is odd. Then let $\hat{x}=\prod_{i=1}^{m} \hat{x}_{i}$, where each $\hat{x}_{i}$ differs from $x_{i}$ by a simple transposition. By Lemma 3.6, $d(x, \hat{x})=2$. Moreover $m-t$ cycles of $\hat{x}$ differ from the corresponding cycle of $y$ by an odd number of simple transpositions. Since $m-t$ is even, we may use the above argument with $x$ replaced by $\hat{x}$ to obtain an element $x^{\prime}$ with $d\left(\hat{x}, x^{\prime}\right) \leq 8$ such that each cycle $x_{i}^{\prime}$ of $x^{\prime}$ differs from the corresponding cycle of $y$ by an even number of simple transpositions. Since $d(x, \hat{x})=2$ we have $d\left(x, x^{\prime}\right) \leq 10$ and as in the case $m \equiv 3 \bmod 4$, each cycle of $x^{\prime}$ differs from the corresponding cycle of $y$ by at most $\left(\begin{array}{c}m-1 \\ 2\end{array}\right)$ simple transpositions.

Corollary 3.7 along with Propositions 3.8 and 3.9 immediately give the next result.

Proposition 3.10. Suppose $x$ has the same orbits as $y$. Then $d(x, y) \leq f(m)$.

Proof of Theorem 1.3 Write $x$ as an $m \times m$ array with the rows being the cycles of $x$. Any permutation which leaves the set of rows fixed produces an element $y$ of $X$ with the same orbits as $x$. Permute the elements of each row in such a way that in each column there is exactly one element of each orbit of $a$. This produces an element $y$ of $X$ with $d(x, y) \leq f(m)$ by Proposition 3.10. Reflecting the array in the main diagonal produces $z \in X$ with $d(y, z)=1$. Now permute the elements of each row of $z$ to produce $v \in X$ 
such that the $i^{\text {th }}$ entry of each row of $v$ is in the $i^{\text {th }}$ orbit of $a$. Again by Proposition $3.10, d(z, v) \leq f(m)$. Finally, reflect again in the main diagonal to product $w \in X$ with $d(v, w)=1$ and the orbits of $w$ are the same as the orbits of $a$. Hence $d(w, a) \leq f(m)$. Therefore $d(x, a) \leq 3 f(m)+2$.

\section{References}

[1] C. Bates, D. Bundy, S. Perkins and P. Rowley. Commuting Involution Graphs for Symmetric Groups, J. Algebra 266(2003), no. 1, 133-153.

[2] C. Bates, D. Bundy, S. Perkins and P. Rowley. Commuting Involution Graphs in Finite Coxeter Groups, J. Group Theory 6(2003), no. 4, 461-476.

[3] C. Bates, D. Bundy, S. Perkins and P. Rowley. Commuting Involution Graphs in Special Linear Groups, Comm. Algebra 32, No.11(2004), 4179-4196.

[4] C. Bates, D. Bundy, S. Hart and P. Rowley. Commuting Involution Graphs in Sporadic Simple Groups, J. Algebra 316, Issue 2(2007), 849-868.

[5] D. Bundy. The Connectivity of Commuting Graphs, J. Comb. Theory, Ser. A 113, Issue 6(2006), 995-1007.

[6] J.J. Cannon and C. Playoust. An Introduction to Algebraic Programming with Magma [draft], Springer-Verlag (1997). 